\section{Objective structural clinical examinations in psychiatry}

OSCEs in Psychiatry. Ed. by Albert Michael. Pp. 210. Illustrated. Elsevier Science Limited. 2004. ISBN 0-443-07297-3.

Objective structured clinical examinations (OSCEs), using real or simulated patients, were developed with a view to improve what some regard as unreliable traditional examination methods by standardising variability in both patients and candidate assessment. As such they are a fairly new phenomenon in the field of psychiatry and have only recently replaced the so-called 'long case' in the UK Royal College of Psychiatrists Part 1 examinations. The aim of this volume is therefore to afford candidates from different countries and medical schools an opportunity to prepare themselves for the range of possible OSCE scenarios that could be encountered when undertaking these examinations.

The volume impresses as well written with information presented in an easy to read format. The list of topics is well chosen and comprehensive, including not only the most important psychiatric scenarios but also a number of allied medical areas such as assessment of ECGs, fundoscopy, resuscitation principles and cranial nerve examination. Although some chapters are not relevant to South Africa (e.g. the UK Mental Health Act), valuable general principles can be garnered from all. Also take special note of the South African contributions!

Owing to the current differences in our own examination proce- 
dures this volume can not strictly be regarded as a study guide for the South African market. However it should prove valuable to a wide range of clinicians, starting from final year medical students, going through to junior psychiatric registrars as well as general practitioners who need to jog their memory with regard to mental health issues. As such I would recommend it as a comprehensive, handy and accessible addition to their bookshelves.

\section{Liezl Koen}

Department of Psychiatry

University of Stellenbosch 
dures this volume can not strictly be regarded as a study guide for the South African market. However it should prove valuable to a wide range of clinicians, starting from final year medical students, going through to junior psychiatric registrars as well as general practitioners who need to jog their memory with regard to mental health issues. As such I would recommend it as a comprehensive, handy and accessible addition to their bookshelves.

\section{Liezl Koen \\ Department of Psychiatry \\ University of Stellenbosch}

\section{Origins of Phobias and Anxiety Disorders: Why More Women Than Men}

Origins of Phobias and Anxiety Disorders: Why More Women Than Men. Michelle G Kraske. Pp. 304. Oxford: Elsevier Ltd. 2003. ISBN 0-08-044032-0.

Anxiety disorders, as we know, are common to both youth and adult populations. This book provides intriguing insights, primarily from a developmental perspective, into the basis of pathological fear and anxiety and explores the question of why a greater risk for anxiety and anxiety disorders exists in women.

Anxiety disorders often precede and predict other disorders (e.g. major depression), suggesting that they may, in fact, represent an early developmental manifestation of shared emotional distress. Once established, these disorders are likely to have a 'scarring' impact, increasing the risk of other disorders that have a similar vulnerability. The book provides a detailed discussion of the broad-based vulnerabilities for anxiety disorders /the salience of negative affect and the threat value of stimuli), and their genetic and environmental contributions, maintaining a developmental focus throughout. In addition, the book details the unique vulnerabilities that are relevant to each of the specific anxiety disorders (panic disorder, social anxiety disorder, post-traumatic stress disorder, specific phobias, and generalized anxiety disorder).

The imperfect relationship between childhood and adult anxiety is highlighted: anxious youths often remain vulnerable to anxiety disorders in adulthood, but 'youth anxiety disorders neither guarantee the development of adult anxiety nor are necessary for adult anxiety'. From a theoretical perspective, the author outlines the pathways that lead to excessive anxiety and fear and draws on up-to-date research from the fields of developmental psychology, neuroscience, cognitive psychology, and psychiatry. A clear and objective analysis follows of how many of the factors that place people at risk for anxiety disorders (e.g. negative affectivity and threat-based style of emotional regulation) are also more strongly associated with being female. Notably, the greater risk for anxiety and anxiety disorders in females progressively diverges from childhood through to adolescence and adulthood. Pertinent to the sex difference is how worry, tendencies to self-focus, greater sensitivity to facial expressions, and reliance on social interpersonal cues in women may underlie higher rates of disorder, as well as more anxious responding (both biologically and socially driven) and higher levels of avoidance behaviour (mediated through sympathetic-adrenal and hypothalamic-pituitary axis activation).

This excellent review is at the cutting edge of its field and is a valuable resource for psychiatrists, psychologists, and other mental health professionals who have often asked the question why some people develop anxiety disorders and others do not, and who would like to gain a more comprehensive understanding of the origins of fear and anxiety.

\section{S Seedat}

MRC Unit on Anxiety and Stress Disorders

Department of Psychiatry

Stellenbosch University

Tygerberg, WCape
All books reviewed are obtainable from SAMA-HMPG Book Department. Tel: (021) 530-6527, fax: (021) 531-4126, e-mail: books@samedical.org. All orders for SAMA members are subject to a $5 \%$ discount. 